MITSUBISHI ELECTRIC RESEARCH LABORATORIES

http://www.merl.com

\title{
Nonlinear Adaptive Estimation of the State of Charge for Lithium-ion Batteries
}

\author{
Wang, Y.; Fang, H.; Sahinoglu, Z.; Wada, T.; Hara, S.
}

TR2013-109 December 2013

\begin{abstract}
This paper considers State of Charge (SoC) estimation of Lithium-ion battery. Different from various prior art, where estimation is performed based on local linearization of a nonlinear battery model, a nonlinear adaptive observer is proposed to estimate the SoC and the parameters of a simplified but nonlinear battery model. A major advantage of the proposed approach is the possibility to establish the exponential stability of the resultant error dynamics of state and parameter estimation. Simulation validates the effectiveness of the proposed approach.
\end{abstract}

IEEE Conference on Decision and Control (CDC)

This work may not be copied or reproduced in whole or in part for any commercial purpose. Permission to copy in whole or in part without payment of fee is granted for nonprofit educational and research purposes provided that all such whole or partial copies include the following: a notice that such copying is by permission of Mitsubishi Electric Research Laboratories, Inc.; an acknowledgment of the authors and individual contributions to the work; and all applicable portions of the copyright notice. Copying, reproduction, or republishing for any other purpose shall require a license with payment of fee to Mitsubishi Electric Research Laboratories, Inc. All rights reserved. 



\title{
Nonlinear adaptive estimation of the state of charge for Lithium-ion batteries
}

\author{
Yebin Wang, Huazhen Fang, Zafer Sahinoglu, Toshihiro Wada and Satoshi Hara
}

\begin{abstract}
This paper considers State of Charge (SoC) estimation of Lithium-ion battery. Different from various prior art, where estimation is performed based on local linearization of a nonlinear battery model, a nonlinear adaptive observer is proposed to estimate the $\mathrm{SoC}$ and the parameters of a simplified but nonlinear battery model. A major advantage of the proposed approach is the possibility to establish the exponential stability of the resultant error dynamics of state and parameter estimation. Simulation validates the effectiveness of the proposed approach.
\end{abstract}

\section{INTRODUCTION}

Lithium-ion $\left(\mathrm{Li}^{+}\right)$batteries have gained widespread use in numerous applications from consumer electronics to power tools since its commercialization, thanks to the higher capacity but reduced size, superior power performance but longer cycle life [1]. Nowadays battery management systems (BMSs) are used to monitor the battery status and regulate the charging and discharging processes for real-time battery protection and performance improvement [2], [3]. An accurate state of charge (SoC) of the battery, usually defined as the percentage ratio of the present battery capacity to the maximum capacity, is a prerequisite to have a desirable BMS.

The SoC of an battery is difficult to measure, and its accurate estimation is also known as a challenging task. Two straightforward but typical SoC estimation methods are voltage translation and Coulomb counting [3]. Both methods have limitations such as the former requires the battery to rest for a long period and cut off from the external circuit to measure the Open Circuit Voltage (OCV), and the latter suffers cumulative integration errors and noise corruption. It is worth noting that both methods do not exploit explicit battery models.

Recent efforts on the SoC estimation concentrate on model-based approaches to improve accuracy. For instance, equivalent circuit models (ECMs) and extended Kalman filter (EKF) type of approaches have been used extensively to estimate the SoC with approximate dynamic error bounds [4], [5], [6]. Other nonlinear observer design approaches have also been used to construct ECM based nonlinear SoC estimators, including sliding mode observer [7], adaptive model

Y. Wang and Z. Sahinoglu are with Mitsubishi Electric Research Laboratories, 201 Broadway, Cambridge, MA 02139, USA. \{yebinwang,zafer\}@merl.com

H. Fang was an intern with Mitsubishi Electric Research Laboratories, and is with Department of Mechanical and Aerospace Engineering, University of California, San Diego, CA 92093, USA. hzfang@ucsd.edu

T. Wada and S. Hara are with the Advanced Technology R\&D Center, Mitsubishi Electric Corporation, 8-11, Tsukaguchi-honmachi, Amagasaki City, 661-8661, Japan. Wada.Toshihiro@bx.MitsubishiElectric.co.jp, Hara.Satoshi@cb.MitsubishiElectric.co.jp reference observer [8] and Lyapunov-based observer [9] etc. The main disadvantage of ECMs-based estimation approaches is the lack of physical meaning of model parameters thus may not be very useful for battery monitoring.

Another important type of battery models, which is generally in the form of nonlinear partial differential equations (PDEs), are derived based on electrochemical principles describing intercalation and diffusion of lithium ions and conservation of charge within a battery. Such electrochemical models have the merit of ensuring each model parameter to retain a proper physical meaning; on the other hand, they are difficult to handle and often necessitates model simplification or reduction. A linear reduced-order electrochemical model is established in [10], to which the classical KF is employed for the $\mathrm{SoC}$ estimation. In [11], the EKF is implemented to estimate the SoC via a nonlinear ordinary differential equation (ODE) model obtained from PDEs by finite-difference discretization. The unscented Kalman filter (UKF) is used in [12] to avoid model linearization for more accurate SoC estimation. Rather than using the ODE model after simplification, nonlinear SoC estimators are also developed in [13], [14] through direct manipulation of PDEs.

Adaptive SoC estimation, which enables the SoC to be estimated when the model parameters are unavailable, has been discussed for some ECMs and electrochemical models, e.g., [6], [15], [16]. This paper makes new contributions to study of this topic, with the aim of developing an nonlinear adaptive SoC estimator with guaranteed convergence.

\section{A REDUCED-COMPleXity MODEL}

In this section, the working mechanism of $\mathrm{Li}^{+}$batteries is briefly introduced. Then a review of the electrochemical principles based single particle model (SPM) is presented, followed by appropriate model simplification for the purpose of the SoC estimation.

\section{A. The Working Mechanism of $\mathrm{Li}^{+}$Batteries}

A schematic visualization of $\mathrm{a} \mathrm{Li}^{+}$battery is presented in Fig 1(a). The positive electrode is typically made from $\mathrm{Li}$ compounds, e.g., $\mathrm{Li}_{x} \mathrm{Mn}_{2} \mathrm{O}_{4}$ and $\mathrm{Li}_{x} \mathrm{CoO}_{2}$. Small solid particles of the compounds are compressed to form a porous structure. Similarly, the negative electrode, usually containing graphite particles, is also porous. The interstitial pores at both electrodes provide intercalation space, where the $\mathrm{Li}^{+}$ can be moved in and out and stored. The electrolyte contains free ions and is electrically conductive, where the $\mathrm{Li}^{+}$can be transported easily. The separator separates the electrodes apart. It allows the exchange of $\mathrm{Li}^{+}$from one side to the 

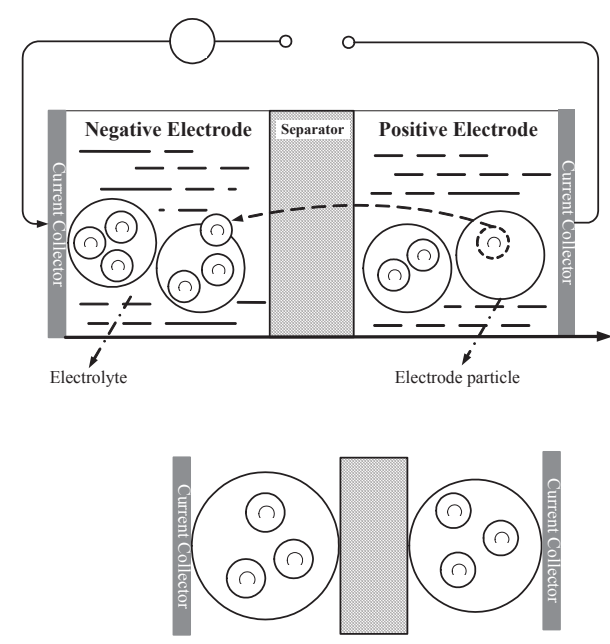

(a)

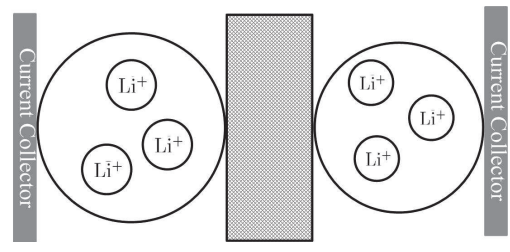

(b)

Fig. 1. Schematic characterization of battery dynamics: (a) the battery charging process; (b) the single-particle model.

other, but prevents electrons from passing through. Electrons are thus forced to flow through the external circuit.

When the battery is being charged, $\mathrm{Li}^{+}$are extracted from particles at the positive electrode into the electrolyte, driven by reaction at the particle/electrolyte interface, and particles at the negative electrode absorbs $\mathrm{Li}^{+}$from the electrolyte. This process not only generates an influx of $\mathrm{Li}^{+}$within the battery, but also builds up a potential difference between the positive and negative electrodes. When it is reversed, the battery is discharging. The chemical reactions in the positive and negative electrodes are, respectively, described by

$$
\begin{aligned}
\mathrm{Li}_{x} \mathrm{Mn}_{2} \mathrm{O}_{4} \underset{\text { discharge }}{\stackrel{\text { charge }}{\rightleftharpoons}} \mathrm{Li}_{x-y} \mathrm{Mn}_{2} \mathrm{O}_{4}+y \mathrm{Li}^{+}+y \mathrm{e}^{-} \\
x \mathrm{Li}^{+}+x \mathrm{e}^{-}+\mathrm{C} \underset{\text { discharge }}{\stackrel{\text { charge }}{\rightleftharpoons}} \mathrm{Li}_{x} \mathrm{C}
\end{aligned}
$$

\section{B. The Single Particle Model}

The single particle model (SPM) simplifies each electrode as a spherical particle with area equivalent to the active area of the electrode [17], [18]. Thus dynamics of $\mathrm{Li}^{+}$in the electrolyte phase are ignored. Although unable to capture all electrochemical processes in batteries, the SPM reduces complexities in identification, estimation and control design to a large extent [11], [14]. To proceed further, a review of the SPM is provided, with the nomenclature shown in Table I.

Input and output of the battery: The external input to the battery is the current $I(t)$ with $I(t)<0$ for charge and $I(t)>0$ for discharge. The terminal voltage is the potential difference

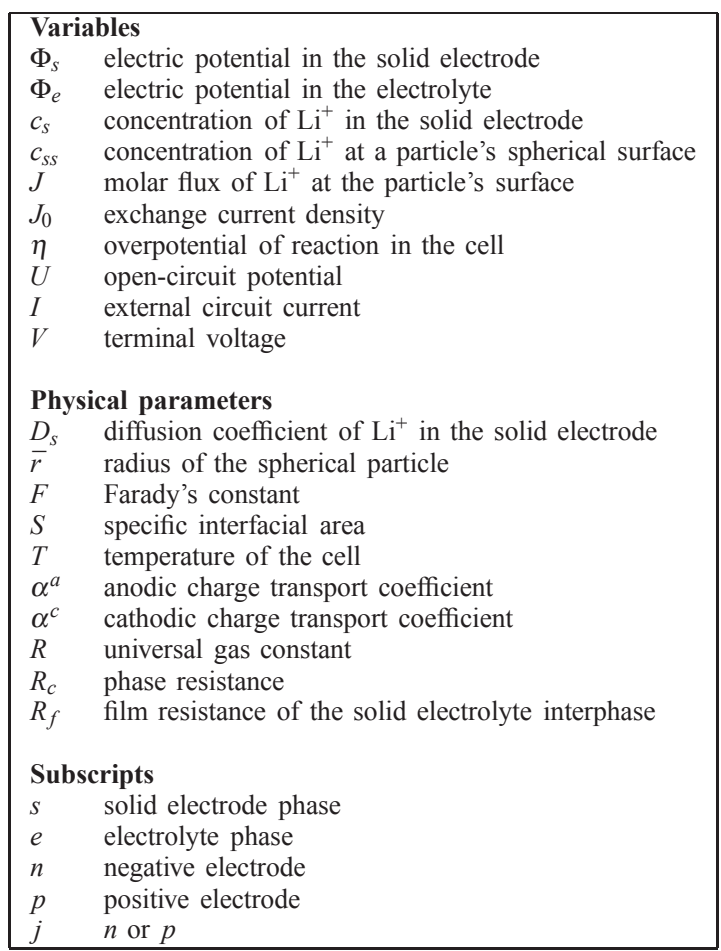

TABLE I

DEFINITIONS AND NOMENCLATURE.

between the two electrodes, that is,

$$
V(t)=\Phi_{s, p}(t)-\Phi_{s, n}(t) .
$$

Conservation of $\mathrm{Li}^{+}$in the electrode phase: The migration of $\mathrm{Li}^{+}$inside a particle is caused by the gradient-induced diffusion. It follows from the Fick's laws of diffusion that

$$
\frac{\partial c_{s, j}(r, t)}{\partial t}=\frac{1}{r^{2}} \frac{\partial}{\partial r}\left(D_{s, j} r^{2} \frac{\partial c_{s, j}(r, t)}{\partial r}\right)
$$

with the initial and boundary conditions given by

$$
c_{s, j}(r, 0)=c_{s}^{0},\left.\quad \frac{\partial c_{s, j}}{\partial r}\right|_{r=0}=0,\left.\quad \frac{\partial c_{s, j}}{\partial r}\right|_{r=\bar{r}_{j}}=-\frac{1}{D_{s, j}} J_{j} .
$$

It is noted that $J_{j}$ is the molar flux at the electrode/electrolyte interface of a single particle. When $j=n$ and $p$, respectively,

$$
J_{n}(t)=\frac{I(t)}{F S_{n}}, \quad J_{p}(t)=-\frac{I(t)}{F S_{p}} .
$$

Electrochemical kinetics: The molar flux $J_{j}$ is governed by the Butler-Volmer equation:

$$
J_{j}(t)=\frac{J_{0, j}}{F}\left[\exp \left(\frac{\alpha_{a} F}{R T} \eta_{j}(t)\right)-\exp \left(-\frac{\alpha_{c} F}{R T} \eta_{j}(t)\right)\right],
$$

where $\eta_{j}(t)=\Phi_{s, j}(t)-\Phi_{e, j}(t)-U\left(c_{s s, j}(t)\right)-F R_{f, j} J_{j}(t)$. The electrolyte phase can be represented by a resistor $R_{c, j}$ in the SPM, implying $\Phi_{c, j}$ can be expressed as $\Phi_{e, j}(t)=$ $R_{c, j} I(t)$. Hence, $\eta_{j}$ becomes

$$
\eta_{j}(t)=\Phi_{s, j}(t)-U\left(c_{s s, j}(t)\right)-F \bar{R}_{j} J_{j}(t),
$$

where $\bar{R}_{j}=R_{c, j}+R_{f, j}$. 
The SPM is represented by (1)-(3), in which $I$ is the external excitation input, $c_{s, j}$ and $\Phi_{s, j}$ are the variables showing the battery status, and $V$ is the model output.

\section{The Reduced Complexity Model}

Average $\mathrm{Li}^{+}$concentration in the electrode phase: Throughout the paper the average concentration of $\mathrm{Li}^{+}$in the particle is considered as the measure of the present battery capacity, or equivalently, the SoC. For an electrode particle, it is defined as

$$
c_{s, j}^{\mathrm{avg}}(t)=\frac{1}{\Omega} \int_{\Omega} c_{s, j}(r, t) \mathrm{d} \Omega,
$$

where $\Omega$ denotes the volume of the particle sphere. From (2), it is obtained that

$$
\begin{aligned}
\dot{c}_{s, j}^{\mathrm{avg}}(t) & =\frac{1}{\Omega} \int_{\Omega} \frac{\partial c_{s, j}(r, t)}{\partial t} \mathrm{~d} \Omega \\
& =\frac{1}{\Omega} \int_{\Omega} \frac{1}{r^{2}} \frac{\partial}{\partial r}\left(D_{s, j} r^{2} \frac{\partial c_{s, j}(r, t)}{\partial r}\right) \mathrm{d} \Omega \\
& =\left.\varepsilon_{j} D_{s, j} \frac{\partial c_{s, j}(r, t)}{\partial r}\right|_{r=\bar{r}_{j}},
\end{aligned}
$$

where $\varepsilon_{j}$ is a constant coefficient. Depending on the electrode polarity, (6) splits into

$$
\begin{aligned}
& \dot{c}_{s, n}^{\mathrm{avg}}(t)=-\frac{\varepsilon_{n}}{F S_{n}} I(t), \\
& \dot{c}_{s, p}^{\mathrm{avg}}(t)=\frac{\varepsilon_{p}}{F S_{p}} I(t) .
\end{aligned}
$$

It is noted from (7)-(8) that the rate of change of $c_{s, j}^{\text {avg }}$ is linearly proportional to the input current $I$. In other words, $c_{s, j}^{\mathrm{avg}}$ is equal to the initial value $c_{s, j}^{\mathrm{avg}}(0)$ plus integration of $I$ over time. This illustrates that the change of SoC depends

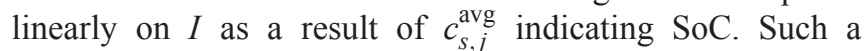
relationship has not only been presented for electrochemical models, e.g., [10], but has also been justified in ECMs, e.g., [5], [19] and the references therein.

Terminal voltage: Suppose there exists a function $\varphi$ such that $c_{s s, j}(t)=\varphi\left(c_{s, j}^{\mathrm{avg}}(t)\right)$ and define $\bar{U}=U \circ \varphi$, where ' $\circ$ ' denotes composition of two functions. Using (4), (1) becomes

$V(t)=\bar{U}\left(c_{s, p}^{\mathrm{avg}}(t)\right)-\bar{U}\left(c_{s, n}^{\mathrm{avg}}(t)\right)+\eta_{p}(t)-\eta_{n}(t)+\left(\bar{R}_{p}-\bar{R}_{n}\right) I(t)$.

With $\alpha_{a}=\alpha_{c}=0.5$, it follows from (3) that

$$
\begin{aligned}
& \eta_{n}(t)=\frac{2 R T}{F} \sinh ^{-1}\left(\frac{J_{n}(t) F}{2 J_{0, n}}\right)=\frac{2 R T}{F} \sinh ^{-1}\left(\frac{\varepsilon_{n} I(t)}{2 J_{0, n}}\right), \\
& \eta_{p}(t)=\frac{2 R T}{F} \sinh ^{-1}\left(\frac{J_{p}(t) F}{2 J_{0, p}}\right)=\frac{2 R T}{F} \sinh ^{-1}\left(-\frac{\varepsilon_{p} I(t)}{2 J_{0, p}}\right) .
\end{aligned}
$$

Thus $V(t)$ becomes

$$
\begin{aligned}
V(t) & =\bar{U}\left(c_{s, p}^{\mathrm{avg}}\right)-\bar{U}\left(c_{s, n}^{\mathrm{avg}}\right) \\
& +\frac{2 R T}{F}\left[\sinh ^{-1}\left(-\frac{\varepsilon_{p} I(t)}{2 J_{0, p}}\right)-\sinh ^{-1}\left(\frac{\varepsilon_{n} I(t)}{2 J_{0, n}}\right)\right] \\
& +\left(\bar{R}_{p}-\bar{R}_{n}\right) I(t) .
\end{aligned}
$$

As such, $V(t)$ consists of two parts. The first is the opencircuit voltage (OCV) that relies on $\bar{U}\left(c_{s, j}^{\mathrm{avg}}\right)$, and the second is the direct feedthrough from $I$ to $V$.
System (7)-(9) provides a concise characterization of the battery dynamics. As aforementioned, $c_{s, j}^{\mathrm{avg}}$ is arguably equivalent to the SoC. Denoting the SoC by a state $x \in[0,1]$, and defining the input $u$ and the output $y$ of the model as the charge current $I$ and the terminal voltage $V$ of the battery, respectively, we have the battery model as follows

$$
\begin{aligned}
\dot{x}(t) & =-\alpha u, \\
y(t) & =h(x)+g(u),
\end{aligned}
$$

where $\alpha$ is a positive parameter, $h(\cdot)$, the part containing $\bar{U}$ in (9), takes the parametric form of $h(x)=\beta_{0} \ln \left(x+\beta_{1}\right)+$ $\beta_{2}$, and $g(\cdot)$ corresponding to the part involving $I$ in (9) are expressed as $g(u)=\gamma_{0}\left[\sinh ^{-1}\left(\gamma_{1} u\right)-\sinh ^{-1}\left(\gamma_{2} u\right)\right]+\gamma_{3} u$, where $\gamma_{i}$ for $i=0,1,2,3$ are from (9).

\section{MAIN RESUlts}

A two-stage approach will be used:

- Stage 1: As $h(\cdot)$ represents the OCV, it is determined using the SoC-OCV data set to identify parameters $\beta_{i}$.

- Stage 2: After $h(\cdot)$ is obtained, the state $x$, and parameters $\alpha, \gamma_{i}$ 's are estimated simultaneously.

The identification in Stage 1 can be formulated as a nonlinear least squares data fitting problem, which can be easily addressed by numerical methods such as Gauss-Newton. The interested reader is referred to [20] for further details. Parameters $\beta_{i}$ are treated as known in Stage 2, where the state and parameter estimation is performed based on [21].

The proposed adaptive observer design in [21] requires the plant in a normal form as follows

$$
\begin{aligned}
& \dot{z}=A z+\varphi(z, u, \theta), \\
& y=C z,
\end{aligned}
$$

where $(A, C)$ is in Brunovsky observer form, $z \in \mathbb{R}^{n}$ is the state vector, $\theta \in \mathbb{R}^{m}$ is the unknown parameter vector, $u \in \mathbb{R}^{s}$ is the input vector, and $\varphi(z, u, \theta)$ has certain triangular dependence on $z$ to enable high gain observer design [22], [23]. Given the plant in the form (11), work [21] also make four assumptions to enable the adaptive observer design. For completeness of this paper, we recite three assumptions (persistent excitation assumption is excluded) as follows.

Assumption 3.1: [21, Assum. (A1)] The state $z(t)$, the control $u(t)$ and the unknown parameters $\theta$ are bounded, i.e., $z(t) \in Z, u(t) \in U$ for $t \geq 0$ and $\theta \in \Omega$ where $Z \in R^{n}, U \in R^{s}$ and $\Omega \in \mathbb{R}^{m}$.

Assumption 3.2: [21, Assum. (A2')] The function $\varphi(z, u, \theta)$ is Lipschitz with respect to $z$ and $\theta$, uniformly in $u$ where $(z, u, \theta) \in Z \times U$.

Assumption 3.3: [21, Assum. (A3')] The nonlinear parameterization function $\varphi(z, u, \cdot)$ is one to one from $\mathbb{R}^{m}$ into $\mathbb{R}^{m}$.

System (10) is clearly not in the form (11) because the output is not a linear function of $z$, thus a state transformation is needed to put (10) into (11). To simplify the transformation, we assume $g(u)$ in system (10) has a linear parametrization. Specifically, we consider the following system

$$
\begin{aligned}
& \dot{x}=\alpha u, \\
& y=\beta_{1} \log \left(x+\beta_{2}\right)+\beta_{3}+\gamma_{1} u,
\end{aligned}
$$


where $x$ is the SoC of a battery, $\beta_{i}$ are known parameters, and $\gamma_{1}, \alpha$ are unknown parameters.

Putting (10) into (11) requires the following parameter dependent transformation

$$
\xi\left(x, u, \gamma_{1}\right)=\beta_{1} \log \left(x+\beta_{2}\right)+\beta_{3}+\gamma_{1} u,
$$

where $\xi$ is the new state variable. We have

$$
\dot{\xi}=\beta_{1} \frac{\alpha}{x+\beta_{2}} u+\gamma_{1} \dot{u}
$$

where $x+\beta_{2}$ is a function of $y, u, \gamma_{1}$ and solved as

$$
x+\beta_{2}=\exp \left(\frac{y-\beta_{3}-\gamma_{1} u}{\beta_{1}}\right)
$$

We rearrange the transformed system and have

$$
\begin{aligned}
& \dot{\xi}=\varphi(y, u, \gamma, \alpha)+\gamma_{1} \dot{u}, \\
& y=\xi
\end{aligned}
$$

where

$$
\phi(\cdot)=\beta_{1} \alpha \exp \left(\frac{\beta_{3}+\gamma_{1} u-y}{\beta_{1}}\right) u .
$$

Remark 3.4: The SoC, represented by $x$, is always positive, also $x+\beta_{2}$ has to be positive if the model (12) is valid. One can verify that the state transformation (13) is a diffeomorphism over $x \in \mathbb{R}^{+}$, i.e., the state transformation (13) is well-defined in the domain where the model (12) is physically meaningful.

The transformed system (14) is in the form of (11), where

$$
\varphi\left(y, u, \dot{u}, \gamma_{1}, \alpha\right)=\beta_{1} \alpha \exp \left(\frac{\beta_{3}+\gamma_{1} u-y}{\beta_{1}}\right) u+\gamma_{1} \dot{u}
$$

The transformed system (14) is nonlinearly parameterized. Clearly the state $\xi$, representing the OCV, external current input $u$, and model parameters $\gamma_{1}, \alpha$ are bounded in a compact set $\mathscr{D}$. Assumption [21, Assum. (A1)] is satisfied. Given $u, \dot{u}, y$ are bounded by a compact set $\mathscr{D}$, the smooth function $\varphi$ is Lipschitz w.r.t. $\xi, \gamma_{1}, \alpha$ and uniformly in $u, \dot{u}, y$. Assumption [21, Assum. (A2')] is also satisfied. Assumption [21, Assum. (A3')] is however not satisfied because given fixed $(\xi, u, \dot{u}, y)$, one can easily find two sets of parameters $\left(\alpha^{1}, \gamma_{1}^{1}\right)$ and $\left(\alpha^{2}, \gamma_{1}^{2}\right)$ such that $\varphi\left(\xi, u, \dot{u}, y, \alpha^{1}, \gamma_{1}^{1}\right)=$ $\varphi\left(\xi, u, \dot{u}, y, \alpha^{2}, \gamma_{1}^{2}\right)$.

Notice that Assumption [21, Assum. (A3')] is not explicitly used to show the convergence, and the proof of Theorem [21, Thm. 4.2] merely relies on Assumptions (A1'),(A2'),(A4'). We may still be able to have a stable adaptive observer as long as Assumption [21, Assum. (A4')] is verified.

We consider the following system

$$
\begin{aligned}
& \dot{\hat{\xi}}=\theta(y-\hat{y})+\hat{\varphi}+\theta \Upsilon \dot{\hat{\rho}} \\
& \dot{\Upsilon}=-\theta \Upsilon+\frac{\partial \hat{\varphi}}{\partial \hat{\rho}} \\
& \dot{\hat{\rho}}=\theta P \Upsilon^{T}(y-\hat{y}) \\
& \dot{P}=-\theta P \Upsilon^{T} \Upsilon P+\theta P
\end{aligned}
$$

where $\rho=\left[\alpha, \gamma_{1}\right]^{T}, \hat{\rho}=\left[\hat{\alpha}, \hat{\gamma}_{1}\right]^{T}, \hat{\varphi}=\varphi(\hat{\xi}, \hat{\alpha}, u)$ and $\theta$ is a sufficiently large positive constant.

Assumption [21, Assum. (A4')], restricted to system (14), is written as follows

Assumption 3.5: The input $u$ is such that for any trajectory of system (15), $\Upsilon(t)$ are persistently exciting i.e., , there exist $\delta_{1}, \delta_{2}, T>0$, for any $t \geq 0$, the following inequalities hold

$$
\delta_{1} I_{2} \leq \int_{t}^{t+T} \Upsilon^{T}(t) \Upsilon(t) \mathrm{d} \tau \leq \delta_{2} I_{2}
$$

where $I_{2}$ is the $2 \times 2$ identity matrix.

Next we verify that Assumption 3.5 may still hold for (15) even Assumption [21, Assum. (A3')] is not satisfied. The $\Upsilon$-dynamics is excited by the following input

$$
\frac{\partial \hat{\varphi}}{\partial \hat{\rho}}=\left[\begin{array}{ll}
\beta_{1} \exp \left(\frac{\beta_{3}+\hat{\gamma}_{1} u-y}{\beta_{1}}\right) u \quad \hat{\alpha} \exp \left(\frac{\beta_{3}+\hat{\gamma}_{1} u-y}{\beta_{1}}\right) u^{2}+\dot{u}
\end{array}\right]
$$

Given $\theta$ a sufficiently large positive constant, we have the approximation $\Upsilon \approx \frac{\partial \hat{\varphi}}{\partial \hat{\rho}}$. Hence, (16) is approximated by

$$
\begin{aligned}
\delta_{1} I_{2} & \leq \int_{t}^{t+T}\left[\begin{array}{ll}
\left(\frac{\partial \hat{\varphi}}{\partial \hat{\alpha}}\right)^{2} & \frac{\partial \hat{\varphi}}{\partial \hat{\alpha}} \frac{\partial \hat{\varphi}}{\partial \hat{\gamma}_{1}} \\
\frac{\partial \hat{\varphi}}{\partial \hat{\alpha}} \frac{\partial \hat{\varphi}}{\partial \hat{\gamma}_{1}} & \left(\frac{\partial \hat{\varphi}}{\partial \hat{\gamma}_{1}}\right)^{2}
\end{array}\right] \mathrm{d} t \\
& =\left[\begin{array}{ll}
\int_{t}^{t+T}\left(\frac{\partial \hat{\varphi}}{\partial \hat{\alpha}}\right)^{2} \mathrm{~d} t & \int_{t}^{t+T} \frac{\partial \hat{\varphi}}{\partial \hat{\alpha}} \frac{\partial \hat{\varphi}}{\partial \hat{\gamma}_{1}} \mathrm{~d} t \\
\int_{t}^{t+T} \frac{\partial \hat{\varphi}}{\partial \hat{\alpha}} \frac{\partial \hat{\varphi}}{\partial \hat{\gamma}_{1}} \mathrm{~d} t & \int_{t}^{t+T}\left(\frac{\partial \hat{\varphi}}{\partial \hat{\gamma}_{1}}\right)^{2} \mathrm{~d} t
\end{array}\right] \leq \delta_{2} I_{2}
\end{aligned}
$$

Since any two square-integrable real-valued functions $\chi_{1}$ and $\chi_{2}$ on an interval $[a, b]$ have an inner product

$$
\left\langle\chi_{1}, \chi_{2}\right\rangle=\int_{a}^{b} \chi_{1}(t) \chi_{2}(t) \mathrm{d} t
$$

if assuming $\partial \hat{\varphi} / \partial \hat{\alpha}$ and $\partial \hat{\varphi} / \partial \hat{\gamma}_{1}$ are integrable over $[t, t+T]$ for any $t \geq 0$, we rewrite (16) as follows

$$
\delta_{1} I_{2} \leq\left[\begin{array}{ll}
\left\langle\frac{\partial \hat{\varphi}}{\partial \hat{\alpha}}, \frac{\partial \hat{\varphi}}{\partial \hat{\alpha}}\right\rangle & \left\langle\frac{\partial \hat{\varphi}}{\partial \hat{\alpha}}, \frac{\partial \hat{\varphi}}{\partial \hat{\gamma}_{1}}\right\rangle \\
\left\langle\frac{\partial \hat{\varphi}}{\partial \hat{\alpha}}, \frac{\partial \hat{\varphi}}{\partial \hat{\gamma}_{1}}\right\rangle & \left\langle\frac{\partial \hat{\varphi}}{\partial \hat{\gamma}_{1}}, \frac{\partial \hat{\varphi}}{\partial \hat{\gamma}_{1}}\right\rangle
\end{array}\right] \leq \delta_{2} I_{2}
$$

We can further verify that the space consisting of all integrable functions over $[t, t+T]$ for any $t \geq 0$ is a pre-Hilbert space and the inner product is a norm of the space, thus the Cauchy-Schwarz inequality holds. Denoting

$$
\|\chi\|^{2}=\langle\chi, \chi\rangle=\int_{t}^{t+T} \chi^{2}(t) \mathrm{d} t
$$

we have

$$
\left\langle\frac{\partial \hat{\varphi}}{\partial \hat{\alpha}}, \frac{\partial \hat{\varphi}}{\partial \hat{\gamma}_{1}}\right\rangle=\left\|\frac{\partial \hat{\varphi}}{\partial \hat{\alpha}} \frac{\partial \hat{\varphi}}{\partial \hat{\gamma}_{1}}\right\| \leq\left\|\frac{\partial \hat{\varphi}}{\partial \hat{\alpha}}\right\| \times\left\|\frac{\partial \hat{\varphi}}{\partial \hat{\gamma}_{1}}\right\| .
$$

That is to say, given $\|\partial \hat{\varphi} / \partial \hat{\alpha}\|$ and $\left\|\partial \hat{\varphi} / \partial \hat{\gamma}_{1}\right\|$ nonzero, the matrix in (17) is not positive definite if and only if $\forall t \geq 0$,

$$
\frac{\partial \hat{\varphi}(\tau)}{\partial \hat{\alpha}}=k \frac{\partial \hat{\varphi}(\tau)}{\partial \hat{\gamma}_{1}}, \quad \forall k \in R, \tau \in[t, t+T] .
$$

We have the following result on the adaptive observer design for system (14). Proof of Proposition 3.6 is omitted due to its similarity to that of [21, Thm. 4.2]. 
Proposition 3.6: Provided that Assumption 3.5 holds, (15) is an adaptive observer of system (12), where $\theta>0$ is a sufficiently large positive constant.

Remark 3.7: Given Assumption 3.5, the adaptive observer (15) yields an error dynamics of the state and parameter estimation exponentially convergent as long as the initial conditions of adaptive observer (15) and the system (14) belong to the compact set $\mathscr{D}$. The exponential stability of the error dynamics also implies certain robustness.

Remark 3.8: The fact that [21, Assum. (A3')] does not hold for the transformed system (14) partially justifies the use of the two-stage approach for joint state and parameter estimation. For the one-stage approach where $\beta_{i}$ are unknown parameters as well, [21, Assum. (A3')] and the PEC [21, Assum. (A4')] is much more difficult to satisfy.

\section{A Numerical Example}

Consider the model in (10) and assume that there is no mismatch between the model and the true system. The parameters are given as follows: $\alpha=4.7496 \times 10^{-5}, \beta_{1}=$ $1.0480, \beta_{2}=0.2208, \beta_{3}=3.9998, \gamma_{1}=-5 \times 10^{-3}$. Here, the values of $\alpha$ and $\gamma_{1}$ are reckoned according to [17], [18], [24] and may have little applicability to a specific battery. The values of $\beta_{i}$ 's are determined by fitting the SoC-OCV data of the battery by experiments. The input to the model is a sinusoid wave $u=10 \sin (10 t)$. We take $\theta=20$ and the following initial conditions (ICs)

$$
\begin{aligned}
& \xi(0)=0.5 ; \quad \hat{\xi}(0)=0 ; \\
& \Upsilon(0)=(0,0), \quad \hat{\rho}(0)=(0,0)^{T}, \quad P(0)=I_{2} .
\end{aligned}
$$

Simulation results are given in Figures 2 - 4, which show that adaptive observer (15) can provide convergent estimation of the transformed system state and parameters. This further implies the state of the original system (12), or the SoC, can also be estimated asymptotically. Simulation also shows that the error dynamics of the state estimation converges much faster than that of the parameter estimation.
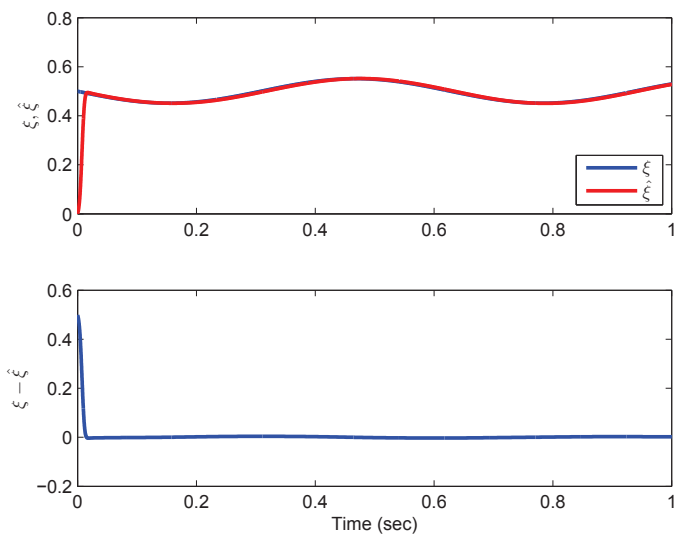

Fig. 2. The transformed system state $\xi$ and its estimation $\hat{\xi}$

We also verify by simulation that the adaptive observer provides convergent estimation of the $\mathrm{SoC}$ and parameters
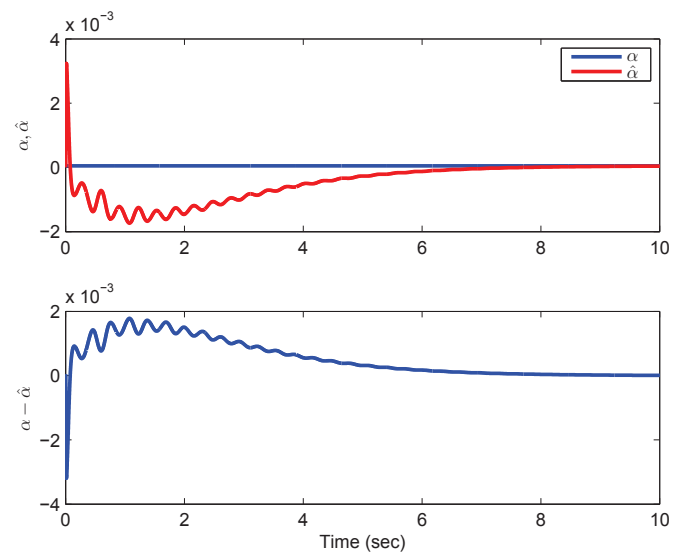

Fig. 3. Parameter $\alpha$ and its estimation $\hat{\alpha}$
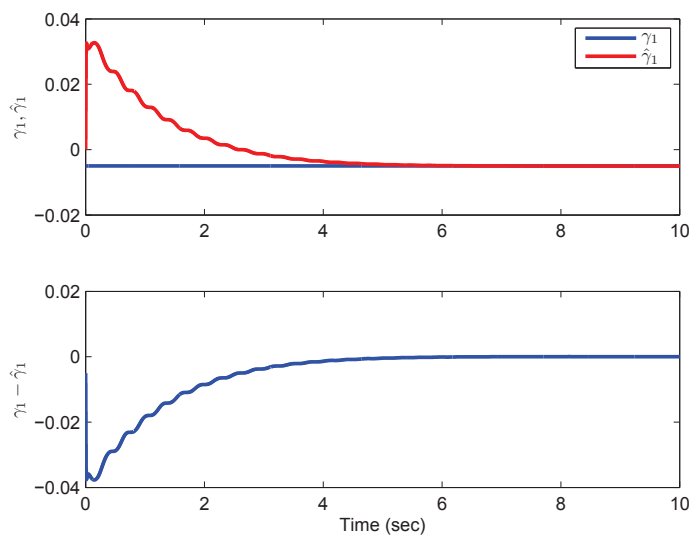

Fig. 4. Parameter $\gamma_{1}$ and its estimation $\hat{\gamma}_{1}$

over a fairly large domain. Given the same input to the model and $\theta$, the adaptive observer (15) is simulated with the following initial conditions (ICs)

$$
\begin{aligned}
& \xi(0)=4 ; \quad \hat{\xi}(0)=100 ; \\
& \Upsilon(0)=(0,0), \quad \hat{\rho}(0)=(0,0)^{T}, \quad P(0)=I_{2} .
\end{aligned}
$$

Simulation results are given in Figures 5 - 7. The estimation error with ICs (19) takes more time to converge than that with ICs (18), but still converges to the true values. Given the ICs (19), extended kalman filter (EKF) or iterated EKF [25] could not provide convergent estimation of the $\mathrm{SoC}$ and parameters.

\section{CONCLUSion}

This paper considered the State of Charge (SoC) estimation of Lithium-ion batteries. A nonlinear adaptive observer was proposed to estimate the $\mathrm{SoC}$ and the parameters of a simplified battery model. The resultant error dynamics of the state parameter estimation is exponentially convergent provided that persistent excitation condition holds. Simulation validated the effectiveness of the proposed approach. 


\section{REFERENCES}

[1] Y. Nishi, "Lithium ion secondary batteries; past 10 years and the future," Journal of Power Sources, vol. 100, no. 1-2, pp. 101-106, 2001.

[2] N. Chaturvedi, R. Klein, J. Christensen, J. Ahmed, and A. Kojic, "Algorithms for advanced battery-management systems," IEEE Control Systems Magazine, vol. 30, no. 3, pp. 49-68, 2010.
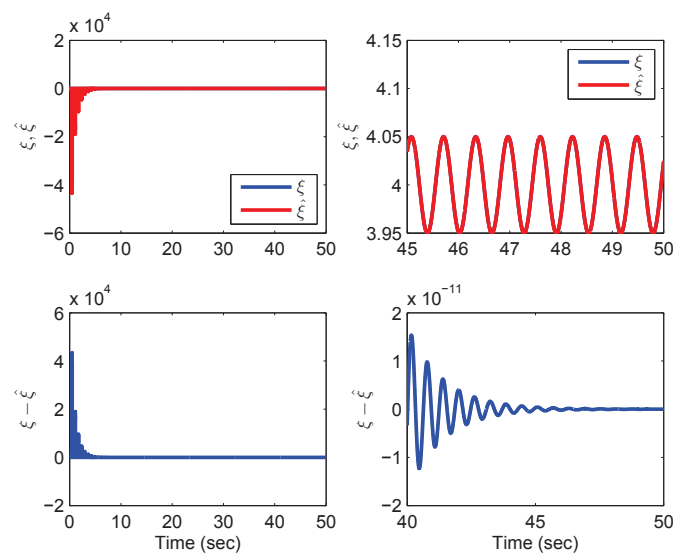

Fig. 5. The transformed system state $\xi$ and its estimation $\hat{\xi}$
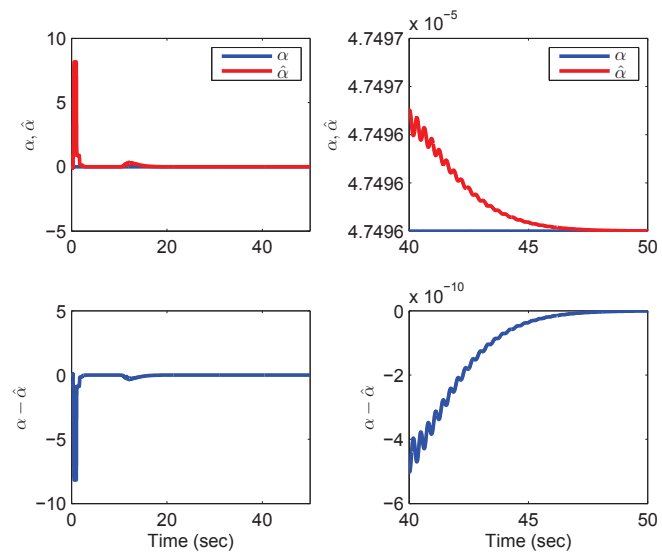

Fig. 6. Parameter $\alpha$ and its estimation $\hat{\alpha}$
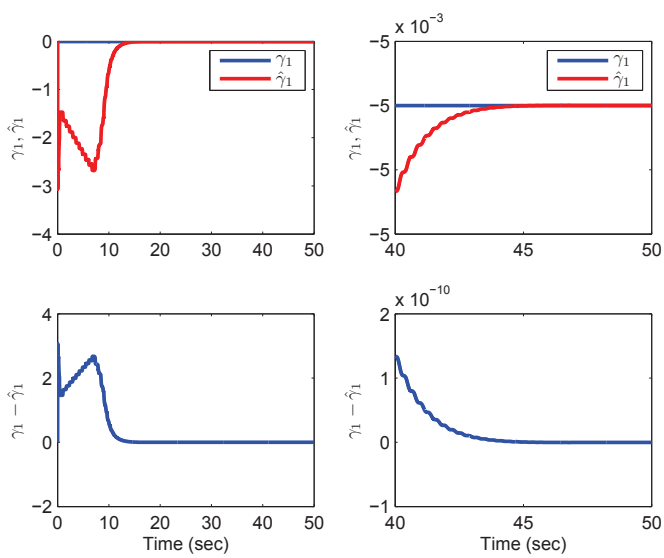

Fig. 7. Parameter $\gamma_{1}$ and its estimation $\hat{\gamma}_{1}$
[3] V. Pop, H. J. Bergveld, P. H. L. Notten, and P. P. L. Regtien, "State-ofthe-art of battery state-of-charge determination," Measurement Science and Technology, vol. 16, no. 12, pp. R93-R110, 2005.

[4] J. Chiasson and B. Vairamohan, "Estimating the state of charge of a battery," IEEE Transactions on Control Systems Technology, vol. 13, no. 3, pp. 465-470, 2006.

[5] G. L. Plett, "Extended Kalman filtering for battery management systems of LiPB-based HEV battery packs: Part 3. state and parameter estimation," Journal of Power Sources, vol. 134, no. 2, pp. 277-292, 2004.

[6] _ _ "Sigma-point Kalman filtering for battery management systems of LiPB-based HEV battery packs: Part 2: Simultaneous state and parameter estimation," Journal of Power Sources, vol. 161, no. 2, pp. $1369-1384,2006$

[7] I.-S. Kim, "The novel state of charge estimation method for lithium battery using sliding mode observer," Journal of Power Sources, vol. 163 , no. 1, pp. 584-590, 2006.

[8] M. Verbrugge and E. Tate, "Adaptive state of charge algorithm for nickel metal hydride batteries including hysteresis phenomena," Journal of Power Sources, vol. 126, no. 1-2, pp. 236-249, 2004.

[9] Y. Hu and S. Yurkovich, "Battery cell state-of-charge estimation using linear parameter varying system techniques," Journal of Power Sources, vol. 198, pp. 338-350, 2012.

[10] K. A. Smith, C. D. Rahn, and C.-Y. Wang, "Model-based electrochemical estimation of lithium-ion batteries," in Proc. IEEE International Conference on Control Applications, 2008, pp. 714-719.

[11] D. Domenico, G. Di Fiengo, and A. Stefanopoulou, "Lithium-ion battery state of charge estimation with a Kalman filter based on a electrochemical model," in Proc. IEEE International Conference on Control Applications, 2008, pp. 702-707.

[12] S. Santhanagopalan and R. E. White, "State of charge estimation using an unscented filter for high power lithium ion cells," International Journal of Energy Research, vol. 34, no. 2, pp. 152-163, 2010.

[13] R. Klein, N. A. Chaturvedi, J. Christensen, J. Ahmed, R. Findeisen, and A. Kojic, "Electrochemical model based observer design for a lithium-ion battery," IEEE Transactions on Control Systems Technology

[14] S. J. Moura, N. A. Chaturvedi, and M. Krstic, "PDE estimation techniques for advanced battery management systems - Part I: SOC estimation," in Proc. American Control Conference, 2012, pp. 559565 .

[15] O. Barbarisi, F. Vasca, and L. Glielmo, "State of charge Kalman filter estimator for automotive batteries," Control Engineering Practice, vol. 14, no. 3, pp. 267-275, 2006.

[16] M. McIntyre, T. Burg, D. Dawson, and B. Xian, "Adaptive state of charge (SOC) estimator for a battery," in Proc. American Control Conference, 2006, pp. 5740-5744.

[17] S. Santhanagopalan, Q. Guo, P. Ramadass, and R. E. White, "Review of models for predicting the cycling performance of lithium ion batteries," Journal of Power Sources, vol. 156, no. 2, pp. 620-628, 2006

[18] M. Guo, G. Sikha, and R. E. White, "Single-particle model for a lithium-ion cell: Thermal behavior," Journal of The Electrochemical Society, vol. 158, no. 2, pp. A122-A132, 2011.

[19] M. Coleman, C. K. Lee, C. Zhu, and W. G. Hurley, "State-ofCharge determination from EMF voltage estimation: Using impedance, terminal voltage, and current for lead-acid and lithium-ion batteries," IEEE Transactions on Industrial Electronics, vol. 54, no. 5, pp. 2550 2557, 2007.

[20] G. A. F. Seber and C. J. Wild, Nonlinear Regression. Wiley, 2003.

[21] M. Farza, M. M'Saad, T. Maatoug, and M. Kamoun, "Adaptive observers for nonlinearly parameterized class of noninear systems," Automatica, vol. 45, no. 10, pp. 2292-2299, Oct. 2009.

[22] G. Bornard and H. Hammouri, "A high gain observer for a class of uniformly observable systems," in Proc. 30th CDC, Brighton, England, 1991, pp. 1494-1496.

[23] J. P. Gauthier, H. Hammouri, and S. Othman, "A simple observer for nonlinear systems - applications to bioreactors," IEEE Trans. Automat. Contr., vol. AC-37, no. 6, pp. 875-880, Jun. 1992.

[24] "Solid-state diffusion limitations on pulse operation of a lithium ion cell for hybrid electric vehicles," Journal of Power Sources, vol. 161, no. 1, pp. 628-639, 2006.

[25] H. Fang, Y. Wang, Z. Sahinoglu, T. Wada, and S. Hara, "Adaptive robust estimation of state of charge for lithium-ion batteries," in Proc. American Control Conference, Washington, DC, 2013, pp. 3491-3497. 